\title{
Wie und wie stark beeinflussen moralische Argumente wirtschaftliches Handeln?
}

\author{
Martin Schröder
}

\begin{abstract}
Zusammenfassung: Die „Corporate Social Responsibility“-Literatur argumentiert mehrheitlich, dass Unternehmen sich sozial verantwortlich verhalten sollten. Darüber vernachlässigt sie die empirische Frage, unter welchen Umständen sie dies tun. Der Artikel versucht, diese Forschungslücke zu schließen, indem er anhand einer repräsentativen Umfrage zeigt, dass Unternehmen moralische Argumente als gewichtige Barriere von Produktionsverlagerungen in Niedriglohnländer nennen und mit geringerer Wahrscheinlichkeit ihre Produktion verlagern, wenn sie moralische Bedenken gegenüber diesem Vorgehen äußern. In einer Fallstudie geht der Artikel der Frage nach, welche Mechanismen diesen Zusammenhang bedingen. Er arbeitet drei Mechanismen heraus, mittels derer moralische Argumente Unternehmen beeinflussen: Erstens beeinflussen sie unter Ungewissheit, was Unternehmen als wirtschaftlich rational definieren. Zweitens beeinflussen moralische Argumente Unternehmen, indem sie deren Sozialkapital angreifen. Drittens beeinflussen sie Unternehmen, indem sie deren öffentliche Legitimität beschädigen. Der Aufsatz schlägt diese drei Mechanismen als Analyseraster vor, mit dem man untersuchen kann, wie moralische Argumente auf Unternehmen wirken.
\end{abstract}

Schlüsselwörter: Soziale Mechanismen · Moralische Argumente $\cdot$ Corporate Social Responsibility $\cdot$ Organisationssoziologie $\cdot$ Legitimität

\section{How and to what degree do moral arguments influence economic action?}

\begin{abstract}
While the corporate social responsibility literature tends to argue that companies should behave socially responsibly, it neglects the empirical question under which conditions they do so. This article fills this research gap by showing under which conditions moral arguments influence companies. The paper uses a representative company survey to show that companies name moral arguments as a strong deterrent to the offshoring of production to low cost countries. In addition, companies are less likely to offshore production when they mention moral scruples against doing so. The article uses a case study to process-trace the mechanisms, by which moral arguments exert this influence. First, it shows how moral arguments influence what is defined as economically rational under uncertainty, thereby influencing economic strategies. Secondly, the article shows how moral arguments influence companies by destroying their social capital, and
\end{abstract}

Online publiziert: 29.06 .2013

(C) Springer Fachmedien Wiesbaden 2013

M. Schröder $(\bowtie)$

Institut für Soziologie, Philipps-Universität Marburg,

Ketzerbach 11, 35037 Marburg, Deutschland

E-Mail: martin.schroeder@uni-marburg.de 
thirdly, it shows how moral arguments can destroy public legitimacy, again influencing economic behavior. The article proposes these three mechanisms as a general framework through which one can understand the influence of moral arguments on companies.

Keywords: Social mechanisms - Moral arguments - Corporate social responsibility · Organizational sociology $\cdot$ Legitimacy

\section{Quelle influence ont les arguments d'ordre moral sur l'activité économique?}

Résumé: La plupart de la littérature consacrée à la responsabilité sociale des entreprises avance l'idée que les entreprises devraient se comporter de manière socialement responsable. Cependant, elle néglige la question empirique de savoir dans quelles conditions exactes les entreprises le font. Cet article comble cette lacune en montrant à partir d'une enquête représentative que les entreprises déclarent que les arguments d'ordre moral sont un obstacle important à la délocalisation de leur production dans des pays à faibles salaires et qu'elles ont moins de chances de délocaliser leur production quand elles émettent des réserves d'ordre moral à ce sujet. Cet article met ensuite en évidence les mécanismes à l'origine de cette corrélation à l'aide d'une étude de cas. Il distingue trois mécanismes par lesquels les arguments d'ordre moral influencent les entreprises. Premièrement, les arguments d'ordre moral influencent ce que les entreprises définissent comme économiquement rationnel dans des situations d'incertitude. Deuxièmement, les arguments d'ordre moral influencent les entreprises en affectant leur capital social. Troisièmement, les arguments d'ordre moral influencent les entreprises en portant atteinte à leur légitimité publique. Cet article propose d'utiliser ces trois mécanismes comme grille d'analyse pour analyser la façon dont les arguments d'ordre moral exercent une influence sur les entreprises.

Mots-clés: Mécanismes sociaux · Argument d'ordre moral · Responsabilité sociale des entreprises · Sociologie des organisations · Légitimité

\section{Einleitung}

Wie stark und mittels welcher Mechanismen beeinflussen moralische Argumente wirtschaftliches Handeln? Der vorliegende Artikel geht der Frage nach, wie sehr und unter welchen Umständen Unternehmen sich in der Entscheidung, ihre Produktion in Niedriglohnländer zu verlagern, von moralischen Argumenten beeinflussen lassen. Er dokumentiert in einem ersten Schritt empirisch, dass nicht verlagernde Unternehmen mehr moralische Bedenken gegenüber Produktionsverlagerungen äußern. Der Artikel arbeitet anschließend anhand einer Fallstudie heraus, mittels welcher Mechanismen moralische Argumente wirken, um diesen Effekt hervorzubringen. Drei Mechanismen werden präsentiert, mittels derer moralische Argumente wirtschaftliches Handeln beeinflussen: Erstens, moralische Argumente füllen individuelle Rationalitätslücken unter Ungewissheit; sie können zudem, zweitens, Sozialkapital in Organisationen verringern und, drittens, öffentliche Legitimität entziehen. Über jeden dieser drei Wirkmechanismen können moralische Argumente wirtschaftliches Handeln beeinflussen.

Obwohl die Wirkung moralischer Argumente auf Unternehmen - und breiter gefasst: auf Organisationen - weithin diskutiert wird, ist die Frage, mittels welcher Mechanismen moralische Argumente wirken, bisher weitgehend unbeantwortet geblieben. Zwar argumentiert eine umfangreiche Literatur, dass es für Unternehmen zunehmend profitabel 
werde, im Einklang mit weithin geäußerten moralischen Argumenten zu handeln - oder zumindest so zu tun -, da Menschen sich mit wachsendem Wohlstand in ihren Konsumentscheidungen zunehmend nach moralischen Kriterien richten würden (vgl. die Überblicksartikel von Fourcade und Healy 2007 sowie von King und Pearce 2010). Doch während diese Forschung und die damit verbundene „Corporate Social Responsibility“Literatur argumentieren, dass Unternehmen sich aus diesem Grunde sozial verantwortlich verhalten sollten, lässt der derzeitige Stand der Forschung die Frage offen, wie moralische Argumente tatsächlich auf Unternehmen wirken (vgl. für diese Kritik Margolis und Walsh 2003, S. 273; Healy 2004, S. 400; Vaara et al. 2006, S. 792; Shamir 2008, S. 12 ff.).

Vorhandene Studien analysieren zwar, wie Aktivisten über internationale Institutionen versuchen, Unternehmen mit moralischen Argumenten zu beeinflussen (Keck und Sikkink 1998, S. 16), wie moralischer Druck als Ausgangspunkt von „Corporate Social Responsibility“-Initiativen fungiert (vgl. Hiß 2006, S. 275 ff.; Marquis et al. 2007) und wie die Missachtung moralischer Proteste Kosten verursacht (Luders 2006; King und Soule 2007; King 2008). Doch die bislang vorliegenden Studien beantworten nicht die Frage, mittels welcher Mechanismen gesellschaftlicher Druck dazu führt, dass Unternehmen ihr Handeln an gesellschaftliche Forderungen anpassen. Etliche Forscher bemängeln diese Forschungslücke (King und Soule 2007, S. 413 f., 438; Vaara et al. 2006, S. 789). Offen bleibt deshalb, unter welchen Umständen moralische Argumente entscheidende Individuen beeinflussen und wann sie über eine Mobilisierung der Belegschaft oder Öffentlichkeit wirken. Auch ist unklar, wie stark moralische Argumente Entscheidungen beeinflussen und unter welchen Umständen sie wirken. Der nachfolgende Artikel sucht nach Antworten auf diese Fragen und beginnt mit der Frage, unter welchen Umständen moralische Bedenken anzutreffen sind und welche Effekte sie haben.

\section{Wie stark wirken moralische Argumente?}

Um eine Antwort auf die Frage zu geben, unter welchen Umständen moralische Bedenken in Unternehmen anzutreffen sind, nutzt der Artikel eine repräsentative Erhebung des Statistischen Bundesamts ${ }^{1}$ aus dem Jahr 2007, das Unternehmen befragte, wie relevant „wirtschaftsethische Probleme (zum Beispiel soziale Verantwortung, Corporate Citi-

1 Die Umfrage richtete sich an die Geschäftsführer oder den jeweils für die Produktionsverlagerung zuständigen Mitarbeiter. Das Bundesamt befragte alle Unternehmen der ,gewerblichen Wirtschaft des Produzierenden Gewerbes [...] und des Dienstleistungssektors mit Ausnahme des Kredit- und Versicherungsgewerbes“ (Zwania 2008, S.481). Insgesamt wurden 20.719 Unternehmen angeschrieben, die über 100 Mitarbeiter hatten. Auf die Befragung antworteten $45 \%$ der Unternehmen, die Rücklaufquote der ausgefüllten Fragebögen lag bei 39\% (zu den Details vgl. ebd., S. 481 f.). Gefragt wurde nach 1) Aspekten der Verlagerung wirtschaftlicher Aktivitäten (Motivationsfaktoren, die Verlagerungen vorantreiben; Barrieren, die Verlagerungen erschweren; Zugehörigkeit zu einer Unternehmensgruppe); 2) Verlagerungserfahrung und -plänen (Zeitplan für verlagernde Aktivitäten, Zielregionen, Kooperationspartner); 3) Auswirkungen der Verlagerung wirtschaftlicher Aktivitäten ins Ausland (Auswirkungen auf das Unternehmen, Auswirkungen auf die Arbeitsplätze, Arbeitsmarkteffekte) und 4) der zukünftigen Entwicklung der Verlagerung wirtschaftlicher Aktivitäten (Statistisches Bundesamt 2008, S. 8). 
zenship)“ als „Barriere bei der Verlagerung wirtschaftlicher Aktivitäten“ wirkten, so die Formulierung im Fragebogen. Diskussionen über die Verlagerung von Produktion ins Ausland sind besonders geeignet, um zu rekonstruieren, wie und wie stark moralische Argumente wirtschaftliches Handeln beeinflussen. Denn Verlagerungsentscheidungen treffen die Belegschaft des verlagernden Unternehmens; heimische Arbeitsplätze werden abgebaut, um Profite zu erhöhen. In solchen Situationen müssen Unternehmen ihr Handeln legitimieren, sie müssen es moralisch rechtfertigen. Diese Rechtfertigungen kritisieren Arbeitnehmervertreter wiederum, um für ihre eigenen Interessen zu werben. Dabei kommt es zu grundlegenden Diskussionen darüber, was Unternehmen erlaubt ist und was nicht. An diesen kann man exemplarisch beobachten, mittels welcher Mechanismen moralischer Druck auf Unternehmen einwirkt (vgl. Soule 2009, S. 59).

Deskriptiv zeigt die Umfrage des Statistischen Bundesamtes, dass genau 50\% der befragten Unternehmen wirtschaftsethische Probleme als sehr relevante oder relevante Verlagerungsbarriere nennen. Moralische Bedenken werden insgesamt sogar etwas öfter als Verlagerungsbarriere angeführt als unzureichendes Know-how, die Abwesenheit geeigneter Zulieferer im Ausland, Zölle, Gefahr von Patentrechtsverletzungen und Unsicherheit über internationale Standards; bei letzteren ist allerdings der Anteil der Antwort „sehr relevant" höher (Statistisches Bundesamt 2008, S. 10). Zumindest in anonymen Umfragen geben Unternehmen somit an, dass moralische Argumente ihr wirtschaftliches Handeln stark beeinflussen. Doch diese Antworten sind möglicherweise dadurch verfälscht, dass Unternehmen sich auch in einer anonymen Umfrage nicht von dem Eindruck lösen können, es sei sozial erwünscht, moralische Bedenken anzugeben, sodass sie diese überhäufig äußern. Eine Möglichkeit, diesem Problem zu entgehen, ist zu untersuchen, wie verschiedene Unternehmenscharakteristika - darunter auch die tatsächliche Neigung, die Produktion zu verlagern - mit den geäußerten moralischen Bedenken gegenüber Produktionsverlagerungen einhergehen. Dies kann zeigen, ob die Nennung moralischer Probleme gegenüber einer Verlagerung neben anderen Einflussfaktoren mit der Tatsache einhergeht, dass ein Unternehmen tatsächlich weniger verlagert. Das Statistische Bundesamt wurde deshalb um eine entsprechende Sonderauswertung ${ }^{2}$ in Form einer logistischen Regression gebeten, die zeigen sollte, unter welchen Umständen die befragten Unternehmen wirtschaftsethische Probleme als Barriere bei der Verlagerung wirtschaftlicher Aktivitäten (so die Bezeichnung des Original-Items) nennen und ob die Nennung moralischer Bedenken mit tatsächlichen Verlagerungswahrscheinlichkeiten einhergeht. Die unten aufgeführten unabhängigen Variablen wurden nach einigen Testläufen aufgenommen, um zu verstehen, was mit moralischen Bedenken einhergeht.

Unternehmen, in denen jemand für das Unternehmenshandeln persönlich verantwortlich ist (mit eigenem Vermögen haftet), könnten stärkere Bindungen an ihren Heimatstandort haben als Unternehmen, die schon aufgrund ihrer Finanzierung keinen Adressaten für moralische Appelle bieten, etwa wenn der Unternehmensbesitz über Aktien verstreut

2 Da die Unternehmensdaten Rückschlüsse auf einzelne Unternehmen zulassen, diese aber ihre Angaben unter der Zusicherung der Anonymisierung machten, war es nicht möglich, die Daten direkt selbst zu bearbeiten. 
ist. Daher untersucht eine erste Variablengruppe, ob Personengesellschaften ${ }^{3}$ mit höherer Wahrscheinlichkeit moralische Bedenken als Verlagerungsbarriere angeben als 1) GmbHs, 2) Aktienunternehmen, 3) Unternehmen mit einer sonstigen privaten Rechtsform, 4) Unternehmen mit einer öffentlichen Rechtsform und 5) Unternehmen mit einer unbekannten Rechtsform. Zudem könnten stärker sozial eingebettete Unternehmen eher moralische Bedenken gegenüber Verlagerungen angeben. Eine zweite Variablengruppe erfasst deshalb, ob 1) Auslandstöchter von Unternehmen, 2) in Deutschland heimische Unternehmen und 3) Unternehmen, die zur Unternehmensgruppenzugehörigkeit keine Angaben gemacht haben, eher wirtschaftsethische Probleme bei Verlagerungen angeben als Unternehmen, die in keinem Unternehmensverbund stehen. Möglich ist auch, dass Unternehmen, die aus nur einem Betrieb bestehen, an ihrem Heimatstandort stärker verwurzelt sind. Schließlich ist es der einzige Standort, den sie haben, sodass diese Unternehmen eher moralische Bedenken als Verlagerungsbarriere anführen könnten. Eine dritte Variablengruppe testet darum, ob Einbetriebsunternehmen eher moralische Bedenken nennen als 1) Mehrbetriebsunternehmen, 2) Mehrländerunternehmen, 3) Betriebe, die Teil eines Mehrländerunternehmens sind, oder 4) Betriebe, die Teil eines Mehrbetriebsunternehmens sind. Denkbar ist darüber hinaus, dass kleinere Unternehmen aufgrund persönlicherer Bindungen zu ihren Arbeitnehmern eher moralische Bedenken nennen. Da größere Unternehmen sich stärker öffentlich rechtfertigen müssen, könnte es aber auch sein, dass diese eher moralische Bedenken als Verlagerungsbarriere angeben. Eine vierte Variablengruppe untersucht deshalb, ob Unternehmen der kleinsten verfügbaren Größenkategorie (mit 100 bis 249 Beschäftigten) eher moralische Bedenken als Verlagerungsbarriere nennen als Unternehmen mit 1) 250 bis 499 Beschäftigten, 2) 500 bis 999 Beschäftigten und 3) 1.000 und mehr Beschäftigten. Möglich ist schließlich auch, dass jüngere Unternehmen weniger moralische Probleme mit einer Verlagerung haben, da in diesen noch keine ,traditionale“ Wirtschaftsethik ${ }^{4} \mathrm{Fuß}$ fassen konnte. Eine fünfte Variablengruppe soll daher zeigen, ob Unternehmen des ,übrigen verarbeitenden Gewerbes“ gegenüber Unternehmen der 1) Hightech-Industrie, 2) wissensintensiver Dienste und 3) ,übriger Wirtschaftsbereiche“ eher moralische Probleme als Verlagerungsbarriere angeben. Zusätzlich kontrollieren zwei Variablen, ob Unternehmen nicht nur deswegen keine moralischen Probleme mit einer Verlagerung angeben, weil sie diese noch nicht durchführten oder nicht planen. Dies prüft auch, ob und wie stark moralische Verlagerungsbedenken mit der tatsächlichen Verlagerungswahrscheinlichkeit einhergehen. Zwei weitere Variablen untersuchen, ob eine Verlagerung zu einem Abbau von Arbeitsplätzen führte. Ohne Kontrolle dieser Variablen wäre es denkbar, dass Unternehmen moralische

3 Offene Handelsgesellschaften, Kommanditgesellschaften sowie alle weiteren Unternehmen, bei denen ein Einzelinhaber oder mehrere Personen mit ihrem Privatvermögen haften.

4 Eine traditionale Wirtschaftsethik orientiert sich gegenüber einer kapitalistischen Wirtschaftsethik daran, ,genug“ Profit zu machen, statt diesen zu maximieren. Man kann argumentieren, dass diese Wirtschaftsethik eher in jenen Unternehmen zu finden ist, in denen sich soziale Bindungen etablieren konnten, was Zeit benötigt (Weber 1980, S. 22, 1988, S. 44 ff.). 
Gründe lediglich deswegen nicht als Verlagerungsbarriere nennen, da mit einer Produktionsverlagerung keine Arbeitsplatzverluste einhergingen. ${ }^{5}$

Ähnlich wie in der Grundgesamtheit der Umfrage nannte etwas mehr als die Hälfte der Unternehmen (4.060 von insgesamt 7.731), für die ausreichend Daten für die logistische Regression verfügbar waren, ,wirtschaftsethische Probleme“ als relevante oder sehr relevante Verlagerungsbarriere. Die in Tab. 1 aufgeführten Odds-Ratios ${ }^{6}$ zeigen, wie stark die verschiedenen Variablen auf die Chance einwirken, dass ein Unternehmen zu der Gruppe gehört, die moralische Bedenken als Verlagerungsbarriere angibt.

Vier unabhängige Variablen weisen eine statistisch signifikante Beziehung zur abhängigen Variablen auf. Unternehmen, die schon Verlagerungen durchführten, weisen eine um 23,7\% verringerte Chance auf, zur Gruppe der Unternehmen zu gehören, die wirtschaftsethische Bedenken äußert. Unternehmen, die Verlagerungen planen, weisen eine um 30,5\% geringere Chance auf, zur Gruppe der Unternehmen zu gehören, die angibt, damit moralische Probleme zu haben. ${ }^{7}$ Zudem weisen Mehrländerunternehmen gegenüber Einbetriebsunternehmen eine um 19,4\% geringere Chance auf, wirtschaftsethische Probleme als Verlagerungsbarriere anzuführen. Unternehmen der Hightech-Industrie weisen gegenüber dem übrigen verarbeitenden Gewerbe eine um 14,4\% verringerte Chance auf, wirtschaftsethische Probleme mit Verlagerungen anzugeben. ${ }^{8}$

Der stärkste Zusammenhang ist somit, dass Unternehmen moralische Probleme mit einer Verlagerung angeben, wenn sie tatsächlich nicht verlagern. Insofern scheint es einen Zusammenhang zwischen moralischen Bedenken gegenüber einer Verlagerung und tatsächlichen Verlagerungen zu geben. Doch mittels welcher Mechanismen könnten moralische Argumente auf Unternehmenshandeln einwirken, sodass es zu diesem Zusammenhang zwischen geäußerten moralischen Bedenken gegenüber einer Verlage-

5 Leider brachte die Umfrage des Statistischen Bundesamtes die Profitabilität der Unternehmen nicht in Erfahrung, weshalb diese Variable fehlt.

6 Der Odds-Ratio von 0,806 (neben „Mehrländerunternehmen vs. Einbetriebsunternehmen“) bedeutet beispielsweise, dass Mehrländerunternehmen gegenüber Einbetriebsunternehmen eine um 19,4\% verringerte Chance haben, zu der Gruppe von Unternehmen zu gehören, die wirtschaftsethische Probleme als Verlagerungsbarriere angibt.

7 Dieses Ergebnis ähnelt einer von Bauer und Hardock (2003, S. 276 ff.) durchgeführten Untersuchung, die $21 \%\left(\mathrm{r}^{2}\right)$ der Verlagerungsvarianz von Unternehmen über den Indikator „Verbundenheit mit dem Inland" erklären konnte.

8 Dass keine der restlichen Kategorien statistisch signifikant ist, kann auch an den verfügbaren Kategorien liegen. So ist zwar denkbar, dass Familienunternehmen eher moralische Probleme mit einer Verlagerung haben. Doch auch eine Aktiengesellschaft (die hier als Rechtsform operationalisiert werden konnte) kann in Familienbesitz sein. Ob ein Unternehmen Teil eines Unternehmensverbundes ist, beeinflusst ebenfalls nicht die Chance, moralische Bedenken anzuführen. Ebenso hat die Beschäftigtengröße keinen Einfluss. Möglich ist, dass sich dabei zwei Effekte gegenseitig ausbalancieren. Einerseits könnten Unternehmen mit weniger Beschäftigten eine engere, persönlichere Bindung zwischen Geschäftsleitung und Belegschaft aufweisen. Andererseits müssen sich größere Unternehmen möglicherweise stärker öffentlich rechtfertigen. Beide Effekte könnten dazu führen, dass ethische Bedenken als Verlagerungsbarriere angegeben werden, sodass es zu keinem eindeutigen Zusammenhang kommt. Insignifikant sind auch die Variablen, die kontrollieren, ob Unternehmen nur deswegen keine Probleme mit einer Verlagerung haben, weil es zu keinem Arbeitsplatzabbau kam. 
Tab. 1: Logistische Regression von Einflüssen auf moralische Bedenken

\begin{tabular}{|c|c|c|c|c|c|}
\hline & & Odds-Ratio & $\begin{array}{l}95 \% \mathrm{~W} \\
\text { Confide }\end{array}$ & dimits & $\begin{array}{l}\text { Signifikanz } \\
(\mathrm{Pr}>\mathrm{ChiSq})\end{array}$ \\
\hline \multirow{5}{*}{$\begin{array}{l}\text { Moralische Beden- } \\
\text { ken in Personenge- } \\
\text { sellschaften vs. }\end{array}$} & GmbHs & 0,904 & 0,726 & 1,126 & 0,3676 \\
\hline & $\begin{array}{l}\text { AGs beziehungsweise } \\
\text { KGaAs }\end{array}$ & 1,019 & 0,767 & 1,353 & 0,8983 \\
\hline & $\begin{array}{l}\text { Unternehmen mit sonst. } \\
\text { priv. Rechtsformen }\end{array}$ & 1,087 & 0,75 & 1,575 & 0,6606 \\
\hline & $\begin{array}{l}\text { Unternehmen mit } \\
\text { öffentlichen Rechtsformen }\end{array}$ & 0,876 & 0,455 & 1,684 & 0,6912 \\
\hline & $\begin{array}{l}\text { Unternehmen mit } \\
\text { unbekannten Rechtformen }\end{array}$ & 1,623 & 0,143 & 18,355 & 0,6956 \\
\hline \multirow{3}{*}{$\begin{array}{l}\text { Moralische Be- } \\
\text { denken in Unter- } \\
\text { nehmen, die nicht } \\
\text { Teil eines Unter- } \\
\text { nehmensverbunds } \\
\text { sind, vs. }\end{array}$} & Auslandstöchtern & 1,142 & 0,998 & 1,307 & 0,0535 \\
\hline & heimischen Unternehmen & 1,057 & 0,954 & 1,171 & 0,2861 \\
\hline & $\begin{array}{l}\text { Unternehmen, die keine } \\
\text { Angabe machten }\end{array}$ & 2,404 & 0,632 & 9,143 & 0,1982 \\
\hline \multirow{4}{*}{$\begin{array}{l}\text { Moralische Beden- } \\
\text { ken in Einbetriebs- } \\
\text { unternehmen vs. }\end{array}$} & Mehrbetriebsunternehmen & 0,948 & 0,823 & 1,092 & 0,4585 \\
\hline & Mehrländerunternehmen & $0,806^{*}$ & 0,721 & 0,902 & 0,0002 \\
\hline & $\begin{array}{l}\text { Betrieben eines Mehrbe- } \\
\text { reichsunternehmens }\end{array}$ & 0,187 & 0,013 & 2,583 & 0,2105 \\
\hline & $\begin{array}{l}\text { Betrieben eines } \\
\text { Mehrländerunternehmens }\end{array}$ & 0,475 & 0,04 & 5,67 & 0,5565 \\
\hline \multirow{3}{*}{$\begin{array}{l}\text { Moralische Beden- } \\
\text { ken in Unternehmen } \\
\text { mit } 100 \text { bis } 249 \\
\text { Mitarbeitern vs. }\end{array}$} & 250 bis 499 Mitarbeitern & 1,036 & 0,921 & 1,165 & 0,5585 \\
\hline & 500 bis 999 Mitarbeitern & 1,160 & 0,979 & 1,375 & 0,0867 \\
\hline & $\begin{array}{l}1000 \text { und mehr } \\
\text { Mitarbeitern }\end{array}$ & 1,162 & 0,933 & 1,446 & 0,1802 \\
\hline \multirow{3}{*}{$\begin{array}{l}\text { Moralische Be- } \\
\text { denken im übrigen } \\
\text { verarbeitenden } \\
\text { Gewerbe vs. }\end{array}$} & $\begin{array}{l}\text { Unternehmen der } \\
\text { Hightech-Industrie }\end{array}$ & $0,856^{*}$ & 0,758 & 0,967 & 0,0122 \\
\hline & $\begin{array}{l}\text { Unternehmen wissensin- } \\
\text { tensiver Dienste }\end{array}$ & 0,955 & 0,784 & 1,163 & 0,6458 \\
\hline & $\begin{array}{l}\text { Unternehmen übriger } \\
\text { Wirtschaftsbereiche }\end{array}$ & 0,908 & 0,811 & 1,016 & 0,0927 \\
\hline \multirow{3}{*}{$\begin{array}{l}\text { Moralische Beden- } \\
\text { ken in Unterneh- } \\
\text { men, die noch nicht } \\
\text { verlagert haben, vs. }\end{array}$} & $\begin{array}{l}\text { Unternehmen, die ver- } \\
\text { lagert haben }\end{array}$ & $0,763^{*}$ & 0,641 & 0,909 & 0,0024 \\
\hline & $\begin{array}{l}\text { Unternehmen, die eine } \\
\text { Verlagerung planen }\end{array}$ & $0,695^{*}$ & 0,559 & 0,864 & 0,0011 \\
\hline & $\begin{array}{l}\text { Unternehmen, die keine } \\
\text { Angabe machten }\end{array}$ & 0,753 & 0,415 & 1,367 & 0,3517 \\
\hline \multirow{2}{*}{$\begin{array}{l}\text { Moralische Be- } \\
\text { denken in Unter- } \\
\text { nehmen, in denen } \\
\text { Verlagerungen } \\
\text { Arbeitsplätze ge- } \\
\text { schaffen haben, vs. }\end{array}$} & $\begin{array}{l}\text { Unternehmen, in denen } \\
\text { Verlagerungen keine } \\
\text { Arbeitsplatzeffekte hatten }\end{array}$ & 1,138 & 0,919 & 1,409 & 0,2352 \\
\hline & $\begin{array}{l}\text { Unternehmen, in denen } \\
\text { Arbeitsplätze verlagert } \\
\text { wurden }\end{array}$ & 0,961 & 0,766 & 1,206 & 0,7335 \\
\hline
\end{tabular}

*Signifikant auf,01-Niveau; $N=7731$ 
rung und deren tatsächlicher Wahrscheinlichkeit kommt? Diese Mechanismen moralischer Einflussnahme, verstanden als ,Prozesse, die bestimmte Ursachen mit bestimmten Wirkungen verbinden“ (Mayntz 2002, S. 24; Hedström und Swedberg 1998), werden in den nachfolgenden Abschnitten qualitativ untersucht.

\section{Wie wirken moralische Argumente?}

Um Mechanismen herauszuarbeiten, die zwei Variablen verbinden, ist das detaillierte „process tracing“ von Fallstudien die optimale Herangehensweise (George und Bennett 2005, S. 214). Deshalb nutzt diese Untersuchung die rekonstruierende Methodik klassischer Unternehmensanalysen (vgl. Gouldner 1954; Blau 1969). Der Artikel analysiert eine tatsächlich stattgefundene Diskussion in einem Unternehmen über Produktionsverlagerung, um zu verstehen, wie moralische Argumente eine wirtschaftliche Entscheidung beeinflussten. Das dazu herangezogene Unternehmen Fernlich ist eine Fallstudie im Sinne eines ,least-likely“-Forschungsdesigns (vgl. Eckstein 1975, S. 119, sowie „crucial case“ in Gerring 2007, S. 115 ff.). Über den Vorstandsvorsitzenden des Unternehmens, Heinrich Zalohma, schrieb eine Tageszeitung, so deutlich wie er ,bekenne sich niemand zu Verlagerungen in Niedriglohnstandorte“. Die Frage stellt sich also: Welche Mechanismen moralischer Einflussnahme werden in solch einem Fall von den Akteuren genutzt, um wirtschaftliches Handeln $\mathrm{zu}$ beeinflussen? Wann funktionieren und wann versagen diese Mechanismen?

Um diese Fragen zu beantworten, rekonstruierte das Forschungsprojekt zuerst die Rahmendaten der Diskussion um Produktionsverlagerung. Es analysierte Memos der Beteiligten, Zeitungsartikel, Flugblätter und Pressemitteilungen. In einem zweiten Schritt wurden die relevanten Teilnehmer interviewt, um sich dem subjektiven Sinn ihres Handelns anzunähern. Im Sinne einer verstehenden Soziologie stand nicht lediglich das, was geschah, im Vordergrund, sondern die Frage, warum es geschah (Weber 1980, S. 1; vgl. Geertz 1973). Dies zu verstehen wird dadurch erschwert, dass Handelnde die letzten Beweggründe ihres Handelns oft selbst nicht nennen können (Weber 1980, S.4; Abel 1948). Das Forschungsprojekt wollte darum nicht wissen, wie Individuen in ihrem eigenen Denken moralische Argumente abwägen. Anstatt solch eines psychologisierenden Zugangs rekonstruierte es mittels einer Prozessanalyse, wie moralische Argumente als soziale Sachverhalte den Verhandlungsverlauf dokumentierbar beeinflussten (vgl. methodologisch Hall 2002, 2007). Zu jedem kritischen Punkt wurden im Verlauf der Untersuchung zwei entgegengestellte Parteien befragt, in der Regel Arbeitnehmervertreter und Geschäftsleitung, um abzugleichen, welche Schilderungen subjektive Interpretation und welche intersubjektiver Konsens waren (vgl. zur Metholodogie der Interviews Gläser und Laudel 2004). Die dabei aufgestellten Hypothesen wurden im Sinne der Grounded Theory immer wieder mit der Empirie abgeglichen, bis eine theoretische Sättigung erreicht war, zusätzliche Empirie also mit den bis dahin herausgearbeiteten Mechanismen gefasst werden konnte (vgl. Glaser und Strauss 1967; Strauss und Corbin 1990). In einem letzten Schritt wurde die Fallstudie an die Beteiligten zurückgeschickt, um mit ihnen die Interpretation der Forscher abzugleichen, welche ansonsten nur das „Konstrukt eines Konstrukts“" wäre (Schütz 1971, S. 5 ff.). Da von den Informanten vertrauliche Informa- 
tionen verlangt wurden, wurden alle Namen anonymisiert. Quellenangaben, die Rückschlüsse auf Personen zulassen, wurden entfernt. Alle Quellen wurden jedoch archiviert und sind prinzipiell einsehbar. Alle Interviews wurden aufgezeichnet, archiviert und sind im Text mit der entsprechenden Zeitmarke, an der etwas gesagt wurde, zitiert. Was also passierte im Unternehmen Fernlich?

Fernlich beschäftigte zum Zeitpunkt der Verlagerungsdiskussion über 50.000 Arbeitnehmer an mehr als sechzig Standorten, davon über 30.000 Arbeitnehmer in mehr als zehn deutschen Werken. Fernlich vergrößerte weltweit seine Belegschaft, während die Anzahl seiner deutschen Arbeitnehmer stagnierte. Das Unternehmen war in seiner Branche überdurchschnittlich erfolgreich; von 1994 bis 2006 verdreifachte sich sein Umsatz, die Umsatzrendite (Ebit) stieg von 4 auf $11 \%$; das Eigenkapital stieg von 2001 bis 2006 von 14 auf $40 \%$ des Umsatzes an; der Aktienkurs verfünffachte sich. Als 2001 die Rendite jedoch vorübergehend einbrach, löste Heinrich Zalohma den bisherigen Vorstandsvorsitzenden ab (Fernlich 3. Interview: 48. Min.). Zeitungen beschrieben Zalohma als „uneitel, klar strukturiert und frei heraus“; er stelle hohe Ansprüche an sich und andere. ${ }^{9}$ Er weigerte sich, einen großen Dienstwagen zu fahren und bei Bahnfahrten die erste Klasse zu nutzen. Dazu kommentierte er: „Wie könnte ich sonst von meinen Leuten verlangen, länger zu arbeiten?“ (Zeitungsartikel) Obwohl sein Jahresgehalt mit 1,5 Mio.€ unter dem vergleichbarer Vorstände lag, nannte er es ,angemessen“, denn ,langfristig kann Geld sowieso nicht motivieren. [...] Das geht nur über das Erreichen von Zielen“ (Zeitungsartikel). Zalohma äußerte, Einsparungen seien wichtiger als Technologieführerschaft, denn jeder Innovationsvorsprung könne innerhalb eines Jahres aufgeholt werden (Ansprache Hauptversammlung). Darum müsse Fernlich seine Preise jährlich um fünf Prozent senken (Fernlich 3. Interview: 14. Min.). Ein Analyst schrieb über Zalohma, er „würde sich sofort selbst wegrationalisieren, wenn er glaubt, dass das dem Unternehmen Geld spart" (Zeitungsartikel). Ein Gewerkschaftsvertreter formulierte, Zalohma sei von seinen Äußerungen völlig überzeugt und setze sie darum ohne Rücksicht auf Kritik um (Fernlich 5. Interview: 2. Min.).

Als Fernlich in den 1990er Jahren von einem Konkurrenzunternehmen aufgekauft werden sollte, demonstrierte dessen Belegschaft noch für die Selbstständigkeit „ihres“ Unternehmens (Fernlich 4. Interview: 29. Min.). Nachdem Zalohma auf einer Betriebsrätekonferenz jedoch verkündete, Fernlich könne seinen Hauptsitz und die damit verbundenen Arbeitsplätze überall haben, verbündeten sich die Betriebsräte zunehmend mit der Gewerkschaft (Fernlich 4. Interview: 31. Min.). In dem traditionsreichen Unternehmen erwarteten sie, dass die alteingesessene Belegschaft vor Kündigungen sicher sein müsse, solange es nicht um das Überleben des Unternehmens gehe (Fernlich 2. Interview: 55. Min.). Doch Zalohma stellte weitere Forderungen. So äußerte er, die Beschäftigten müssten sich klar werden, dass ,nicht 35 bis 37 Stunden pro Woche die normale Arbeits-

9 Davon nahm er auch den Vorstand nicht aus. Als ein Mitglied des Führungsstabes einmal forderte, mehr als die üblichen 150 Euro pro Hotelübernachtung ausgeben zu dürfen, rügte Zalohma ihn: „Wir verlangen gerade von unseren Mitarbeitern in den Fabriken, dass die von 37 auf 40 Stunden [Arbeitszeit] hochgehen. Und das, was ich [...] ausgebe in einer Luxussuite, dafür müssen fünf bis sechs Arbeiter eine Woche arbeiten? Das kann doch nicht Ihr Ernst sein, dass Sie das hier haben wollen.“ (Fernlich 3. Interview: 87. Min.) 
zeit sind, sondern 43 bis 45 Stunden“ (Zeitungsartikel). Dreimal forderte er, die Arbeitszeit zu flexibilisieren und zu verlängern, jedes Mal stimmte die Gewerkschaft zu - mit wachsendem Unmut. Im Sinne eines sozialen Austauschs (vgl. Blau 1964, S. 94) meinten die Arbeitnehmer, Zalohma habe aufgrund ihrer Zugeständnisse nun reziprok eine moralische Bringschuld, die restlichen Arbeitsplätze zu halten, schließlich seien sie ihm weit entgegengekommen (Fernlich 1. Interview: 1. Min.).

Doch kurze Zeit später teilte der Vorstandsvorsitzende den Arbeitnehmervertretern auf einer Aufsichtsratssitzung überraschend mit, er werde trotz aller Konzessionen den Heimatstandort Taubingen aufgrund unerwarteter Marktentwicklungen schnellstmöglich schließen. Dessen Produktion werde er ins Ausland verlagern und die 320 Arbeitnehmer entlassen. Der Taubinger Betriebsrat Florian Harz protestierte, Zalohma könne die Produktionskapazität nicht verlagern, denn erstens erwirtschafte Taubingen jährlich 20 Mio. Euro Gewinn ${ }^{10}$, zweitens wäre die Schließung ein Rechtsbruch gegenüber dem vor sechs Monaten abgeschlossenen Standortsicherungsvertrag. Zalohma entgegnete, er könne eine wirtschaftlich sinnvolle Entscheidung nicht revidieren, nur weil sie unbequem und nicht jeder mit ihr einverstanden sei. Während ein Spezialprodukt aus Taubingen zwölf Euro koste, könne das günstigste Auslandswerk dieses für acht Euro produzieren. Bei 1,5 Mio. Produkten jährlich könne er somit sechs Millionen Euro einsparen. Nur ein schlechter Kaufmann würde diese Einsparung nicht realisieren. Der Gewerkschaftssprecher argumentierte dagegen, Zalohma dürfe „für eine Zigarettenschachtel“ pro Produkt kein Werk schließen (Fernlich 3. Interview: 8. Min.). Die Arbeitnehmervertreter argumentierten, drastische Einschnitte wären vertretbar, um das Unternehmen vor einer Pleite zu retten, doch zum Zwecke der Gewinnmaximierung seien Zalohmas Pläne nicht hinnehmbar. Durch die Verlagerung wollte Zalohma das Äquivalent von $0,05 \%$ des jährlichen Unternehmensumsatzes und $0,5 \%$ des jährlichen Gewinns einsparen. Darüber entbrannte ein deutschlandweiter Konflikt.

Dieser begann damit, dass Fernlichs Kommunikationsabteilung Zalohma warnte, sein Verlagerungsplan könne zu einem Imagedesaster werden, was die Ersparnis der Verlagerung möglicherweise mehr als aufzehren würde. Doch Zalohma entgegnete, er könne nicht aus Rücksicht auf seinen Ruf von einer wirtschaftlich sinnvollen Entscheidung Abstand nehmen; er müsse schließlich keinen Beliebtheitswettbewerb gewinnen, sondern ein Unternehmen lenken. Der Chef der Kommunikationsabteilung widersprach ihm, es komme nicht auf die tatsächliche wirtschaftliche Angemessenheit seiner Entscheidung an, sondern wie die Öffentlichkeit darauf reagiere, da dies wirtschaftliche Folgen für Fernlich habe. Zalohma antwortete, er sei nicht Vorstandsvorsitzender geworden, um sich betriebswirtschaftliche Entscheidungen von Medien, Betriebsrat und öffentlicher Meinung oktroyieren zu lassen (Fernlich 3. Interview: 7. und 32. Min.).

Er verkündete Taubingens Belegschaft erneut, sie schnellstmöglich entlassen zu wollen. Er erklärte, Fernlich habe bedauerlicherweise Überkapazitäten und müsse die Produktion dort schließen, wo sie am teuersten sei. Leider habe er keine Wahl, als dieser wirtschaftlichen Logik zu folgen. Die Standortvereinbarung, die Kündigungen ausschloss, sei für ihn irrelevant, da er sich „nur an Verträge halte, die ich selbst unterzeichnet habe“

10 Gemessen am Verkaufspreis der Produkte Minus den dafür anfallenden Produktionskosten. 
(Zeitungsartikel). ${ }^{11}$ Zalohma äußerte per Pressemitteilung, soziale Verwerfungen aufgrund seiner Entscheidung seien bedauerlich, doch angesichts wirtschaftlicher Zwänge unvermeidlich, schließlich müsse auch ein erfolgreiches Unternehmen sparen (Fernlich 3. Interview: 2. Min.). Betriebsrat und Gewerkschaft konzedierten zwar, Zalohma könne die Betriebsvereinbarung bei „unvorhersehbarer Marktentwicklung“ tatsächlich kündigen. Davon könne jedoch sechs Monate nach Abschluss der Vereinbarung keine Rede sein. Ganz im Gegenteil sei Fernlichs Aktienwert seit Jahresbeginn um 50\% gestiegen; die betroffene Unternehmenssparte erwirtschafte eine Rendite von 15\% (EBIT). Zwar verweigerte Zalohma die Herausgabe der genauen Werksrendite, doch obwohl Taubingen nur ca. ein Prozent der Produktion ausmachte, erwirtschafteten die dortigen High-EndProdukte $10 \%$ des Unternehmensumsatzes und $20 \%$ des Gewinns. Jedoch konzedierten auch Arbeitnehmervertreter, dass andere Werke noch profitabler als Taubingen seien. Die mit moralischen Argumenten geführte Debatte drehte sich um die Frage, ob ein Unternehmen diese Produktivitätsunterschiede ausnützen dürfe, obschon es hohe Gewinne machte und die Heimatbelegschaft unter der Gewinnmaximierung leiden würde.

Mit dem Argument, Zalohmas Gewinnmaximierung sei moralisch nicht legitim, mobilisierten die Arbeitnehmervertreter die Belegschaft. Auf einer Betriebsversammlung äußerte der Betriebsrat des zu schließenden Werkes: „Gier und ewig steigende Renditeziele sind die einzige Motivation des Vorstands. Der Mensch zählt nichts. Jetzt sollen sogar gewinnträchtige Standorte geschlossen werden. Die Gleichung Kostensenkung gleich Wettbewerbsfähigkeit gleich sichere Arbeitsplätze gilt nicht mehr.“ Daraufhin begannen empörte Arbeiter zu protestieren. Die Gewerkschaft baute vor der Hauptverwaltung eine Bühne auf; Sprechchöre forderten Zalohma ergebnislos auf zu erscheinen (Fernlich 1. Interview: 15. Min.). Der Konflikt eskalierte, da sich unternehmensexterne Akteure auf die Seite der Belegschaft stellten. Der Landesbischof schrieb in einer Zeitung, es gebe eine „ethische Grenze“ des Profitstrebens. Angesichts der Konzessionen der Arbeitnehmer und dem hohen Werksgewinn äußerten Politiker parteiübergreifend, dass Eigentum verpflichte. Zeitungsartikel warfen Zalohma einen „kranken Raubtierkapitalismus" vor. Ein Manager aus einem Konkurrenzunternehmen äußerte, Zalohma stelle sich „vollends ins Abseits“ (Zeitungsartikel). Andere Konkurrenzunternehmen nutzten den Konflikt, indem sie in Zeitungsanzeigen mit dem Argument für Kunden warben, Deutschland im Gegensatz zu Fernlich verbunden zu sein.

Diese Proteste hatten wirtschaftliche Folgen für Fernlich. Händler berichteten, ein Viertel ihrer Kunden boykottiere Fernlichs Produkte. Einige Händler weigerten sich auch von sich aus, diese weiterhin zu führen (Zeitungsartikel). Zalohma kommentierte: „Wenn der Kunde bereit wäre, mehr für das Produkt zu bezahlen, weil es aus Deutschland kommt, könnten wir hier fertigen. Das ist aber nicht so." (Zeitungsartikel) Gewerkschaft und Betriebsrat versuchten nun nicht mehr, Zalohma selbst mit moralischen Appellen zu überzeugen; sie redeten mittlerweile über statt mit ihm. Moralische Argumente sollten Belegschaft und Öffentlichkeit gegen ihn mobilisieren, um sein als unmoralisch deklarierbares Verhalten zu verteuern und damit wirtschaftlich unattraktiv zu machen. Ein Gewerkschaftssprecher erklärte dies retrospektiv folgendermaßen:

11 In der Tat musste der Vorstandsvorsitzende die damals getroffene Vereinbarung nicht unterschreiben, damit diese gültig war. 
„Im Grunde genommen spielte für uns das Unternehmen und die Person an sich in der ganzen Strategie keine Rolle, sondern für uns war wichtig, über die öffentliche Meinung so viel Druck zu machen, dass Zalohma sich überlegt, ob es sinnvoll ist, diesen Krieg am Ende war es ein Krieg - zu Ende zu führen oder ob es nicht besser ist zu versuchen, mit uns einen Kompromiss zu machen. [...] Bilder zu schaffen für die Medien, das war ein wichtiger Teil in der ganzen Strategie.“ (Fernlich 4. Interview: 59. Min.)

Tatsächlich unterstützten die Medien die Interpretationslinie der Gewerkschaften. Der Zeitungsartikel „Zalohma Nimmersatt“ griff das Bild der Raupe Nimmersatt auf, die frisst, bis ihr schlecht wird. Immer wieder tauchte in Zeitungsartikeln das Argument auf, Gewinne seien nur bis zu einem gewissen Punkt moralisch vertretbar und dürften nicht auf dem Rücken der Belegschaft gemacht werden. So fragte eine Tageszeitung: „Warum [...] haben die Beschäftigten Opfer gebracht, wenn Zalohma daraufhin das Fallbeil senkt?“"Weitere Zeitungen schrieben, es stelle sich ,angesichts von Rekord-Bilanzen die Frage nach Zalohmas sozialer Verantwortung“. Dass Zalohma die Rendite erhöhte und die damit verbundenen Konsequenzen ehrlich und frühzeitig mitteilte, rief öffentliche Empörung hervor. Der Journalist einer großen Tageszeitung fragte ihn, wie es sich als „marktradikaler Rambo“ lebe. Zalohma antwortete, für ihn stelle sich trotz Verfünffachung des Aktienwertes in vier Jahren und einer Rendite auf das eingesetzte Kapital von $18 \%$ nicht die Frage, wann Profitstreben unanständig werde. Denn er könne sich nicht auf vergangenen Erfolgen ausruhen, sondern müsse auch weiterhin eine hohe Rendite erarbeiten. Dazu müsse er mitunter Werke schließen, selbst wenn dies der Öffentlichkeit nicht gefalle. Bei nur zehn Prozent deutscher Aktionäre bleibe ihm jedoch keine andere Wahl. Ein kapitalistisches Unternehmen könne keine Arbeitsplatzgarantie geben, da es keine Abnahmegarantie bekomme. Seiner sozialen Verantwortung sei er nachgekommen, indem er den Beschäftigten ihren Arbeitsplatzverlust frühzeitig mitteilte, sodass sie sich darauf einstellen konnten, äußerte er wiederholt in Zeitungsinterviews. ${ }^{12}$ Sein Vorgehen sei zwar hart, doch gerade deswegen sozial verantwortlich, denn es steigere die Unternehmensrendite. Der Gewerkschaft hielt Zalohma vor, lediglich eine nationale Interessengruppe zu sein: „Der Mitarbeiter in Osteuropa kann nicht verstehen, warum wir die Produkte hier bauen [...]. Deshalb sage ich immer wieder: Die ökonomische Moral in diesem Land ist eine sehr lokale. Ich kann und darf keinen Unterschied machen zwischen einem deutschen und einem rumänischen Arbeitsplatz.“ (Zeitungsartikel) Ein weiteres Vorstandsmitglied antwortete gereizt auf die gewerkschaftliche Kritik: „Das heißt, es kratzt niemanden, wenn wir Arbeitsplätze in Osteuropa abbauen?“ (Fernlich 3. Interview: 45. Min.).

Dieses Argument brachte die Gewerkschaft in potenzielle Erklärungsnot. Denn tatsächlich hatten andere Länder die Beschäftigung möglicherweise nötiger als Deutschland. Die Medien ließen sich jedoch nicht auf die Argumente der Geschäftsleitung ein. Sie sympathisierten mit den Gewerkschaften. Obwohl die Geschäftsleitung klarmachte, Eltern mit kleinen Kindern würden nicht gekündigt, porträtierten Reportagen immer wieder zwei Familienväter, die angaben, durch die anstehenden Entlassungen ihre Familien

12 Dieser Sichtweise blieb Zalohma bis zum Ende hin treu. Das Einzige, was er im Nachhinein als Fehler ansah, war, den Standortsicherungsvertrag überhaupt abzuschließen, obwohl er vorher nicht sicher sein konnte, ihn einzuhalten (Fernlich 3. Interview: 44. Min.). 
bald nicht mehr ernähren zu können. Damit unterschlugen Journalisten besseren Wissens Informationen und manipulierten die öffentliche Meinung im Gewerkschaftssinne. Eine Boulevardzeitung schrieb: ,aus dem Manager des Jahres ist der Kotzbrocken des Jahres geworden“. Dass ein anderes Großunternehmen gleichzeitig weitaus mehr Entlassungen als Fernlich plante, thematisierten die Medien in dem Zeitraum kaum. Denn Zalohma hatte sich mit seinen Äußerungen zum perfekten Feindbild gemacht. Die Geschäftsleitung sah sich entsprechend als Opfer einer Gewerkschaftskampagne, die die Öffentlichkeit geschickt über deren moralische Befindlichkeit mobilisierte und wirtschaftlichen Druck ausübte, indem sie das Unternehmensimage schädigte. Obwohl die Öffentlichkeitsabteilung Zalohma genau dies prophezeit hatte, feuerte er mit seinen Äußerungen die Gewerkschaftskampagne weiterhin an, denn er wollte wiederum nicht von seinem Standpunkt ablassen, den er für richtig hielt (Fernlich 3. Interview: 6. Min.).

Fernlichs hohe Rendite wurde von einer Rechtfertigung zu einer Last, die selbst Rechtfertigung verlangte. Der WirtschaftsMinister des Bundeslandes sowie der Ministerpräsident und der Oberbürgermeister baten Zalohma um Vier-Augen-Gespräche. Doch jeder äußerte nach seinem Gespräch, es sei ihm nicht gelungen, Zalohma nahezulegen, dass er einen gewinnbringenden Betrieb nicht schließen dürfe, um den Unternehmensgewinn zu maximieren. In einer Anhörung bekundeten alle Landtagsfraktionen ihre Solidarität mit den Beschäftigten. Eine Partei rief sogar zu einem Kundenboykott auf (Zeitungsartikel). Selbst der Präsident des Arbeitgeberverbandes äußerte, Unternehmen sollten eine Betriebsvereinbarung einhalten, wenn sie es sich leisten könnten, sonst setzen sie ihre Glaubwürdigkeit aufs Spiel. Eine Reportage mit dem Titel „Bosse ohne Moral“ beschrieb Zalohma als „Vertreter einer ausschließlich von der Wall Street geprägten Moral, für die Deutschland irrelevant ist und die an den Grundlagen der Gesellschaft rüttelt“. Taubingens Betriebsrat äußerte: „Wenn wir rote Zahlen schreiben würden, könnte man ja über alles reden. [...] Aber nicht, wenn man gerade einen solchen Riesengewinn gemacht hat" (Zitat Internetartikel). Längst ging es um mehr als die 320 Kündigungen, die nur ein Prozent der deutschen Belegschaft ausmachten. Es ging darum, ob Zalohma die Unternehmensrendite auf Kosten profitabler Arbeitsplätze maximieren durfte - und beide Seiten wurden zunehmend kompromisslos. Der Druck stieg, als die Gewerkschaft einen wichtigen Broker überzeugte, dass der Aktienkurs unter den sozialen Spannungen leiden werde. Eine große Bank kritisierte daraufhin den „negativen Newsflow“ des Unternehmens und empfahl, die Aktie wegen eines ,internen und externen Image- und Glaubwürdigkeitsschadens“ zu verkaufen. Es drohten angeblich Auseinandersetzungen, „die zu Millionen-Schäden führen könnten“ (Zeitungsartikel). Betriebsräte protestierten; selbst der Betriebsrat des ausländischen Werks, das von der Verlagerung profitierte, bekundete seine Solidarität. Doch etliche Beschäftigte trauten sich nicht zu demonstrieren, da die Geschäftsleitung drohte, sie abzumahnen. Die Arbeitnehmervertreter waren unsicher, wie sie mit der verärgerten, jedoch auch eingeschüchterten Belegschaft Druck ausüben sollten (Fernlich 6. Interview: 15. Min.).

Fernlichs Kommunikationsabteilung war unterdessen „,entsetzt über Zalohma, weil er sich an keine Regeln der internen Kommunikation gehalten hat" (Fernlich 6. Interview: 2. Min.). Die Öffentlichkeitsabteilung musste einem Desaster für das Unternehmensimage zusehen, wie einer ihrer Mitarbeiter retrospektiv äußerte: „Das ist, wie wenn Sie sehen, dass da ein Zug auf etwas zufährt und Sie können ihn nicht mehr 
anhalten. Sie kriegen ihn nicht mehr zu stoppen! Dann kommt eine Eigendynamik ans Werk und dann geht das Gesichtwahren los, auf beiden Seiten." (Fernlich 3. Interview: 91. Min.) Die Medien berichteten nicht mehr über die Kampagne der Gewerkschaften; sie wurden ein Teil von ihr. Fernlichs Management führte diese Parteilichkeit darauf zurück, dass die Journalisten Zalohmas Haltung selbst unmoralisch fanden. Die damit verbundene negative Berichterstattung kostete Fernlich laut einer Medienresonanzanalyse seiner Öffentlichkeitsabteilung ca. 1,5 Mio. Euro (Fernlich 3. Interview: 23. Min.). ${ }^{13}$

Aufgrund der öffentlichen Berichterstattung eskalierte der Konflikt auch im Aufsichtsrat. Der Aufsichtsratsvorsitzende äußerte zu Beginn einer Sitzung: „Ich bin heute mit dem Taxi hergekommen, und da schimpft der Taxifahrer eine halbe Stunde über diese Scheißkapitalisten bei Fernlich. Ich hab mich gar nicht getraut zu sagen, wer ich bin.“ (Zitat, durch einen Anwesenden wiedergegeben) Schon vor dem Treffen hatten nicht nur die Gewerkschaften, sondern auch der Landesvorsitzende einer großen Partei alle Aufsichtsratsmitglieder angeschrieben und eine Unternehmenskultur gefordert, die stärker auf die moralischen Befindlichkeiten der Bevölkerung eingehe. Ähnlich argumentierten nun sogar drei Aufsichtsratsmitglieder der Arbeitgeberseite, dass Zalohma nicht jegliches Handeln mit Renditemaximierung legitimieren könne; er habe auch eine gesellschaftliche Verantwortung. Zalohma hatte aufgrund der moralischen Mobilisierung die Aufsichtsratsmehrheit nicht mehr hinter sich. Wie beeinflusste die Öffentlichkeitskampagne die Arbeitgeberseite im Aufsichtsrat? Zwei Anwesende erinnern sich an die entscheidende Aufsichtsratssitzung:

„Die Anteilseigner im Aufsichtsrat [...] haben klar gesagt, dass sie erwarten, dass der Vorstand den Konflikt löst. Die hatten auch die Schnauze voll, da jeden Tag negative Schlagzeilen zu lesen.“ (Zitat eines Anwesenden)

„Das gibt es offensichtlich, dass wir, irgendwie verinnerlicht, ein Modell haben: Sie akzeptieren zwar eine Wirtschaftsweise, die auf Privateigentum und Renditeorientierung beruht, aber haben in quantitativen Margen auch gar nicht ausformulierte Grenzen. Ich glaube, das ist zwar ein schwächer werdender, aber durchaus immer noch vorhandener Zug selbst gestandener Unternehmerpersönlichkeiten. Und in diesem Aufsichtsrat waren es welche. Da gab es welche, die waren Hardliner, die waren auch bis zum Ende durchgängig Hardliner, die sich exponiert haben, allerdings damit auch ein Stück weit isoliert haben. Diese Apologeten eines freien Unternehmertums haben sich da auch isoliert. Und diese rheinische Variante, die hat dann, nennen Sie es Skrupel, nennen Sie es dann doch noch Gewissen gehabt. Die Arbeitnehmerseite hat erfolgreich daran appellieren können. Das ist gelungen." (Zitat eines An.wesenden)

13 Eine Medienresonanzanalyse wertet die Berichterstattung über ein Unternehmen aus. Bei positiver Berichterstattung wird gefragt, wie viel man für Anzeigen ausgeben müsste, die der positiven Berichterstattung vom Umfang her gleichkommen. Bei negativer Berichterstattung wird dieser Wert ins Negative verkehrt. Es handelt sich dabei jedoch um Schätzwerte. Sowohl die Frage, ob positive Berichterstattung einen ähnlichen Effekt hat wie Werbeanzeigen, als auch die Frage, wie stark eines von beiden zum Unternehmensgewinn beiträgt, bleiben nur spekulativ zu beantworten. Zumindest schien das Unternehmen davon auszugehen, dass negative Berichterstattung Kosten verursache. 
Selbst in einem Börsenunternehmen, welches Zalohma wirtschaftlich erfolgreich führte, fand er keine Mehrheit mehr für seine Renditemaximierung. Zusammen mit drei Mitgliedern der Arbeitgeberseite beschlossen die Arbeitnehmervertreter - und damit die Mehrheit des Aufsichtsrates -, dass es keinen Machtkampf zwischen Zalohma und der Gewerkschaft geben dürfe, in dem eine der beiden Seiten dauerhaft beschädigt würde (Fernlich 5. Interview: 20. Min.). Zalohma bot daraufhin erstmals an, Taubingen sechs Monate später zu schließen, damit jedoch immer noch sechs Monate vor Ablauf der Standortvereinbarung. Doch das war den Arbeitnehmervertretern nicht genug; sie lehnten ab. Als nächstes stand eine Weihnachtspause an; Zalohma schätzte Öffentlichkeit und Belegschaft als konfliktmüde ein; er glaubte nicht, dass diese im neuen Jahr erneut mobilisierbar seien. Er lehnte weitere Verhandlungsrunden ebenso ab wie den gewerkschaftlichen Vorschlag, einen Vermittler einzuschalten. Den Widerstand der Gewerkschaften tat er in einem Zeitungsinterview mit der Bemerkung ab: „die Handvoll verbliebener Ideologen werden wir auch noch zur Einsicht bringen“. In einem weiteren Interview verkündete er, er könne seine wirtschaftliche Entscheidung nicht davon abhängig machen, dass er damit in Deutschland einen Beliebtheitswettbewerb gewinne, denn seine ,unternehmerische und soziale Verantwortung“ (Zitat) gelte weltweit. Weiter äußerte er:

„Der Vorstand hat [...] keine Sonderverantwortung speziell für einen deutschen oder amerikanischen Standort. Was, denken Sie, würden die 50.000 ausländischen Mitarbeiter sagen, wenn wir den geplanten Stellenabbau aufgrund des öffentlichen Drucks entgegen unserer wirtschaftlichen Urteilskraft rückgängig machen? Die Lage ist genauso schwierig für die weggefallenen Arbeiter in verschiedenen Auslandsstandorten. International interessiert das Thema niemanden. Die Angelegenheit ist politisch aufgebauscht. Auch falls ich mich wiederhole: Dieser Aufschrei der Entrüstung ist Resultat einer lokalen Moral.“ (Zeitungsartikel)

Zalohma hatte mit seinem Vorwurf der ,,lokalen Moral“ recht. Während deutsche Zeitungen ihn wegen ca. 300 Arbeitsplätzen am Heimatwerk kritisierten, schloss er in den USA ein dreimal so großes Werk. Keine deutsche Zeitung berichtete darüber (Fernlich 3. Interview: 66. Min.). Öffentlich wertete die Gewerkschaft sein Interview als ,Zeichen, dass er kein Interesse an einer Lösung habe“ (Pressemitteilung). Insgeheim begrüßte sie, dass er sich radikal äußerte. Denn in den Worten eines Gewerkschaftssekretärs hatte er der Gewerkschaft damit ,natürlich auch jeden Gefallen getan. Gleich Anfang Januar hatte er ein Interview gegeben, dass er uns auch gar nicht verstehen könnte. Wir wären doch alle nicht ganz richtig im Kopf, und man müsse die 42-Stunden-Woche einführen - und hatte damit natürlich immer wieder Ereignisse geliefert, auf die wir reagieren konnten. Und jedes Mal die Journalisten ... ich habe manchmal das Gefühl gehabt, sie freuten sich, wenn er ein Interview gegeben hat, weil sie genau wussten, jetzt geht der Konflikt noch zwei Wochen weiter und wird auch noch medial von uns begleitet.“ (Fernlich 4. Interview: 64. Min.)

Personaldirektor Stoller übernahm nun die Verhandlungsführung, denn Zalohma trug in den Augen aller Beteiligten nicht zu einer Einigung bei (Fernlich 4. Interview: 89. Min.). Der Vorsitzende der Gewerkschaft schrieb in einem Kommentar, der Konflikt sei „,von grundsätzlicher Bedeutung“, denn Zalohma versuche, ,ungezügeltem Profitstreben Tür und Tor zu öffnen. [...] Wenn Zalohma den selbst beobachteten ,Aufschrei der Empörung‘ abschätzig als ,Resultat einer lokalen Moral‘ bezeichnet, dann zeigt dies, was 
er von der sozialen Marktwirtschaft und dem Sozialstaatsgebot unseres Grundgesetzes hält.“ Anstatt den Vorwurf der „lokalen Moral“ als Kritik anzunehmen, versuchte die Gewerkschaft, die Deutungshoheit darüber zu erlangen, was als angemessene moralische Forderung gelten sollte. Die lokale Moral, die sie vertrete, seien das Sozialstaatsgebot des Grundgesetzes und der sozialen Marktwirtschaft. Dessen Normen, so die Gewerkschaft, gelten auch für Zalohma und insgesamt für wirtschaftliches Handeln. Beide Seiten versuchten, ihre Interessen als deckungsgleich mit dem Allgemeinwohl darzustellen. Zalohma äußerte dagegen, nur wenn Unternehmen hohe Renditen und lange Arbeitszeiten durchsetzen, könne Deutschland wettbewerbsfähig werden. Die Gewerkschaft - und die Medien mit ihr - argumentierten dagegen, der Kapitalismus verliere an Legitimation, wenn Zalohmas Verhalten Schule mache.

Sollte Zalohma nicht binnen einer Woche einlenken, wollte die Gewerkschaft einen Tariftatbestand über Abfindungsregeln schaffen, was ihr einen Streik ermöglichen würde (Fernlich 6. Interview: 80. Min.). Diesen konnte die Gewerkschaft jedoch nur planen, da sie wusste, dass die gesamte deutsche Belegschaft hinter ihr stand. Dass dies der Fall war, ist insofern erstaunlich, als die Kündigungen - in Relation zu den Gesamtarbeitsplätzen - marginal waren. Selbst der gemäßigte DGB rief jedoch angesichts Zalohmas Renditemaximierung zu Protesten auf. Fünfzig Betriebsräte erklärten sich mittlerweile sogar bereit, aus Protest gegen Zalohmas als unmoralisch wahrgenommenes Verhalten ihre Werke durch „Dienst nach Vorschrift“ lahmzulegen (Zeitungsartikel). Zwar war es den Arbeitnehmervertretern bisher nicht gelungen, Aktienwert oder Absatz zweifelsfrei messbar zu senken. Doch nun stufte eine zweite Bank die Fernlich-Aktie von „Buy“ auf „Hold“ ab mit dem Argument, die zunehmenden Konflikte könnten Fernlich hunderte Millionen Euro kosten (Fernlich 2. Interview: 73. Min. und Zeitungsartikel). Zwei Tage später erreichten die Proteste ihren Höhepunkt. Fast die gesamte deutsche Belegschaft demonstrierte, was juristisch fragwürdig war. Da 99 von 100 Demonstranten nicht selbst von den Kündigungen betroffen waren, sind diese Proteste nicht restlos durch rationales Eigeninteresse zu erklären. Die Gewerkschaft äußerte, es gelang ihr vielmehr, die Proteste durch moralische Argumente anzufachen, was wirtschaftlichen Druck ausübte, wie sich ein Gewerkschaftsführer retrospektiv erinnerte:

„Fernlich ist nur nervös zu kriegen, wenn die Aktie anfängt, nervös zu werden. Deswegen haben wir auch die Veranstaltung vor der Hauptversammlung gemacht. Das sind alles Dinge, um den Anleger nervös zu machen: ,Bei Fernlich ist nicht alles Friede, Freude, Eierkuchen.' Und wir wissen, dass natürlich Investoren genau hingucken: ,Wie läuft das denn da, das soziale Verhältnis im Unternehmen?‘ Das ist zwar erst drittrangig oder viertrangig, aber bei der Gesamtbewertung des Unternehmens spielt es eine Rolle.“ (Fernlich 4. Interview: 18. Min.)

Der immer wieder vorgetragene Vorwurf der Gewerkschaft lautete, Kündigungen zur Profitmaximierung seien bei hohen Gewinnen moralisch nicht zu rechtfertigen (Fernlich 1. Interview: 8. Min.). Als annähernd die gesamte Belegschaft protestierte, lenkte die Geschäftsleitung ein. Sie versprach, von betriebsbedingten Kündigungen abzusehen. Bei Schließung des Werks sollten die verbliebenen Arbeitnehmer nun in eine Qualifizierungsgesellschaft wechseln, die sie weiterbilden würde und bei der die Arbeitnehmer im ersten Jahr 90 und im zweiten Jahr 67 \% ihres Gehalts beziehen sollten. Danach könnten alle Belegschaftsmitglieder zu Fernlich zurückkehren oder freiwillig ausscheiden. Arbeit- 
nehmer, die sich entschlossen, das Unternehmen zu verlassen, stattete die Geschäftsleitung mit einem sehr guten Sozialplan aus. Statt der anfänglich angebotenen 9.000 Euro Abfindung zahlte sie pro ausscheidendem Arbeitnehmer durchschnittlich 35.000 Euro. Die Geschäftsleitung beklagte lediglich noch, dass die Gewerkschaft das Doppelte der üblichen Abfindungen forderte, da sie aufgrund der durch moralische Argumente angestachelten Proteste in einer starken Verhandlungsposition war (Fernlich 3. Interview: 34. Min.). Die Geschäftsleitung schloss Taubingen schlussendlich ein Jahr später als ursprünglich geplant mit Auslaufen des Standortsicherungsvertrages (Fernlich 3. Interview: 36. Min.).

Ein anonymer Experte äußerte, durch die angebotenen Weiterbildungs- und Umschulungsprogramme werde Taubingens Schließung ca. 50 Mio.Euro kosten. Dies wären ca. 150.000 Euro pro verlagertem deutschem Arbeitsplatz. Die Geschäftsleitung sprach von ca. 15 Mio. (Fernlich 3. Interview: 79. Min.). Ersteres entsprach der Summe, die die Werkschließung innerhalb von 8,3 Jahren einsparen sollte; Letzteres der Summe, die sie in 2,5 Jahren einsparen sollte. Zu diesen Kosten der Werkschließung kamen noch die Kosten durch Imageschädigung. Obwohl diese schwer zu beziffern sind, äußerte ein Vorstandsmitglied Fernlichs im Nachhinein, die Gewerkschaft habe ihre Karte mit der Medienkampagne „100 Prozent richtig gespielt“ (Fernlich 3. Interview: 42. Min.). Die professionelle Manipulation der Medien habe Fernlich „Millionen gekostet“ (Fernlich 4. Interview: 39. Min.). Teile der Geschäftsleitung äußerten anonym, der Konflikt habe mehr gekostet, als die Verlagerung einsparen sollte; eine Werkschließung mit Auslaufen der Standortvereinbarung hätte die Geschäftsleitung zudem auch ohne Konflikt haben können. Nach dem Konflikt gab Zalohma einem der Betriebsräte die Hand und sagte: „Eins zu null für Sie.“ (Fernlich 2. Interview: 21. Min.)

\section{Drei Mechanismen moralischer Einflussnahme}

In einer repräsentativen Umfrage des Statistischen Bundesamtes nannten $50 \%$ aller Unternehmen moralische Argumente als Verlagerungsbarriere. Eine statistische Analyse dieser Umfragedaten ergab, dass Unternehmen, die entsprechende moralische Bedenken äußern, tatsächlich weniger verlagern. Die obige Fallstudie zeigte exemplarisch, wie es zu diesem Zusammenhang kommen kann, wie also moralische Argumente unternehmerisches Handeln beeinflussen. Der erste Mechanismus moralischer Einflussnahme, der aus der Fallstudie ersichtlich wurde, bestand in der Nutzung moralischer Argumente auf der Mikroebene. Moralische Argumente wurden als Appelle an den entscheidenden Unternehmensakteur gerichtet. Als dies im Falle Zalohmas scheiterte, weitete sich der Konflikt aus. Arbeitnehmervertreter versuchten nun, mit moralischen Argumenten auf der organisationalen Mesoebene die Belegschaft zu beeinflussen, um Druck auszuüben. Als auch das weitgehend scheiterte, wurden moralische Argumente auf der gesellschaftlichen Makroebene in die Öffentlichkeit gebracht und übten dadurch Druck aus. Abbildung 1 fasst diese drei Mechanismen moralischer Einflussnahme zusammen.

Diese drei Mechanismen, mittels derer moralische Argumente wirtschaftliches Handeln beeinflussen, bauen aufeinander auf. Niemand, der versucht, Einfluss zu nehmen, würde einen gesamtgesellschaftlichen Konflikt anzetteln, ohne vorher versucht zu haben, 
Dritter Wirkmechanismus moralischer Argumente: Öffentlichkeitsmobilisierung $\rightarrow$ Makro-Konflikt auf Gesellschaftsebene

Moralische Argumente wirken hier als Waffe, um die Öffentlichkeit zu einem Unternehmensboykott anzuregen, indem Verlagerungspläne zum Zweck der Gewinnmaximierung als unmoralisch präsentiert werden. Auch dies führt zu einer ,strategischen Rekalkulation“" wirtschaftlicher Interessen, insofern moralische Argumente Kosten verursachen.

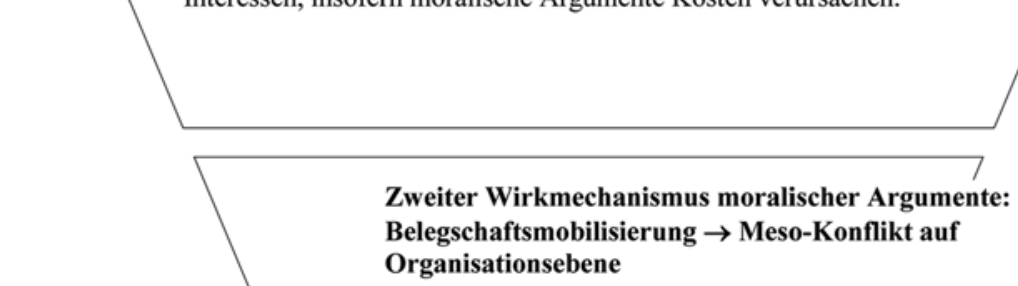

Moralische Argumente wirken hier als Waffe, um die Belegschaft zu demotivieren, indem ihr suggeriert wird, unmoralisch behandelt $\mathrm{zu}$ werden. Wenn dies zu einer sinkenden Motivation und damit zurückgehenden Produktivität der Belegschaft führt und auch so gesehen wird, kommt es zu einer ,strategischen Rekalkulation“ wirtschaftlicher Interessen, insofern moralische Argumente Kosten verursachen.

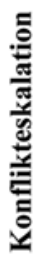

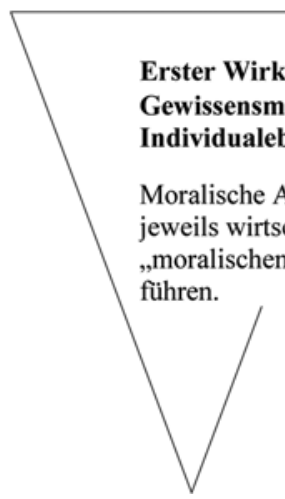

Abb. 1: Einflussmechanismen moralischer Argumente

den Konflikt innerhalb der betreffenden Organisation zu lösen. Ein Konflikt auf Organisationsebene kann wiederum erst entstehen, wenn in direkten Gesprächen mit denjenigen, die die Organisation leiten, kein zufriedenstellendes Ergebnis zustande kommt. Die folgenden Abschnitte ordnen die drei Mechanismen moralischer Einflussnahme in soziologische Theorien ein, um ihre Wirkungsweise und Eskalationsdynamik näher zu charakterisieren. 


\subsection{Wirkmechanismen moralischer Argumente gegenüber Individuen}

In einem ersten Schritt wirken moralische Argumente als Appelle an entscheidende Akteure. Moralische Argumente können versuchen, diese auf Ziele zu verpflichten, die als deckungsgleich mit dem Allgemeininteresse hingestellt werden können. Demgegenüber können Adressaten moralischer Argumente argumentieren, mit ihrem Handeln anderen allgemein akzeptierten Interessen zu dienen. Im vorliegenden Fall argumentierte die Geschäftsleitung, zur Wertsteigerung gegenüber den Aktionären verpflichtet zu sein statt den Interessen der Heimatbelegschaft. Da solch eine Gegenargumentation sich mit gesellschaftlichen (statt auf Eigeninteresse bezogenen) Interessen und Normen rechtfertigt, handelt es sich durchaus um ein moralisches Argument (vgl. für diese Moraldefinition Durkheim 1999, S. 11; 1986, S. 40 f.; 1953, S. 49 f.). Dagegen ist anzunehmen, dass im Fall einer tatsächlich vorliegenden Wahrung von Eigeninteressen diese nur in Grenzfällen als legitime Selbstbereicherung gerechtfertigt werden kann. Wenn dies den gesellschaftlich akzeptablen Normen widerspricht, macht sich eine solche Legitimationsstrategie argumentativ angreifbar.

Ein weiterer und damit verbundener Mechanismus, mittels dessen moralische Argumente auf individueller Ebene wirken, besteht aufgrund von Ungewissheit darüber, was optimale wirtschaftliche Handlungen und Entscheidungen sind. Unter Ungewissheit können moralische Argumente all das als auch wirtschaftlich sinnvoll darstellen, was als moralisch vertretbar gilt (vgl. Beckert 1996). Menschen treffen Entscheidungen unter Ungewissheit innerhalb eines „Frames“, der eine bestimmte Entscheidung - auch bei Abwesenheit objektiver Kalkulierbarkeit - wirtschaftlich rational erscheinen lässt (Kaplan 2008). Moralische Argumente können genau solch einen Frame beeinflussen. Sie wirken in diesem Fall nicht dadurch, dass ihnen der Vorzug gegenüber wirtschaftlichen Rationalitätserwägungen gegeben wird. Vielmehr beeinflussen moralische Argumente unter Ungewissheit, was überhaupt als wirtschaftlich rational definiert wird. Dies muss jedoch nicht bewusst geschehen und tut es wahrscheinlich in der Regel auch nicht. Dieser Wirkmechanismus ist deshalb nur begrenzt wissenschaftlich nachvollziehbar. Im vorliegen Fall war sich der Adressat moralischer Argumente jedoch sicher, genau ökonomisch berechnen zu können, ob sich eine Verlagerung lohnt. Moralische Argumente konterte er deshalb mit der Behauptung, die „Fakten ließen keine andere Wahl“ (Fernlich 2. Interview: 36. Min.; Fernlich 3. Interview: 31. Min.). Insofern bleibt dieser Einflussmechanismus in Situationen unwirksam, in denen die Beteiligten eine Entscheidung als angesichts der Fakten rationalste wahrnehmen. Moralische Argumente können als real wahrgenommene Fakten insofern nicht wegdiskutieren, sie können aber die „Frames“ beeinflussen, nach denen vermeintliche Fakten als tatsächliche Fakten wahrgenommen und bewertet werden.

Auf dieser ersten Ebene wirken moralische Argumente somit, wenn sie zu den Wertoder Rationalitätsvorstellungen desjenigen passen, an den sie adressiert werden. Eine „Rationalitätslücke“, in der moralische Appelle auf die Rahmenvorstellungen von Rationalität einwirken können, öffnet sich, wenn Ungewissheit herrscht, was in einem konkreten Fall wirtschaftlich rational ist. Im obigen Fallbeispiel blieben moralische Appelle an die Entscheidungsträger jedoch deshalb folgenlos, weil diese sie für wirtschaftlich unangebracht hielten und sich in der Überzeugung wiegten, genau berechnen zu können, 
was wirtschaftlich rational und daher zu tun ist. Diese Unversöhnlichkeit der Geltungsansprüche - Moral auf der einen, Ökonomie auf der anderen Seite - führte zur Eskalation des Konflikts auf einer zweiten Ebene. Moralische Argumente begannen, auf der Organisationsebene zu wirken.

\subsection{Wirkmechanismen moralischer Argumente in Organisationen}

Nachdem moralische Appelle an den verantwortlichen Entscheidungsträger wirkungslos blieben, sprachen die Nutzer dieser Appelle nicht mehr mit, sondern über den Adressaten der Appelle. Zuerst argumentierten sie organisationsintern, dass der Adressat moralischer Appelle Normen und Interessen der Arbeitnehmer nicht berücksichtige. Daraufhin begannen Arbeitnehmer, der Geschäftsleitung ihr Vertrauen zu entziehen, was wirtschaftlich kostspielig war. So äußerte ein Mitglied des Aufsichtsrates: „Die brauchen heute, um was zu verhandeln, dreimal so lange, wie sie vorher gebraucht haben“ (Fernlich 4. Interview 24. Min.; vgl. auch Fernlich 3. Interview: 26. Min.). Moralische Argumente können somit auf der Organisationsebene Druck ausüben, indem sie implizite Verträge beschädigen. Diese in etlichen Studien dokumentierten impliziten Verträge bewirken, dass Arbeitnehmer in einer Organisation mehr als „Dienst nach Vorschrift“ leisten, wohingegen Arbeitgeber eine Rücksichtnahme üben, die über streng rechtliche Voraussetzungen hinausgeht (Durkheim 1977, S. 267 ff.; Weber 1980, S. 28; Gouldner 1954, S. 137, 179; Blau 1964, S.206; Granovetter 1985, S. 487 ff.). Diese gegenseitige Zusammenarbeit über das vertraglich geregelte Maß hinaus, welche rechtlich kaum einklagbar ist und auf Vertrauen beruht, kann man als das Sozialkapital einer Organisation konzeptualisieren (Putnam 1993, S. 35 f.). Moralische Argumente wirken somit in Organisationen über den Mechanismus, dass sie bisherige Kooperationspartner als unmoralisch, weil unkooperativ darstellen - und damit als nicht mehr vertrauenswürdig. Indem moralische Argumente dadurch eine wirtschaftlich und sozial effiziente Kooperation in einer Organisation beeinträchtigen, können sie wirtschaftlichen Druck aufbauen. In dem hier geschilderten Fall war dieser Einflussmechanismus jedoch wirkungslos, da eine seiner Vorbedingungen nicht gegeben war. Zalohma meinte, er könne seine Belegschaft am besten durch Druck und Überwachung motivieren, sodass er deren gesunkene Kooperationswilligkeit nicht bedauerte (Fernlich 3. Interview: 17. Min.; Fernlich 4. Interview: 45. Min.). Dieser Einflussmechanismus scheint somit nur zu wirken, wenn die Zerstörung des Sozialkapitals und die Verringerung der Kooperationsbereitschaft auch von denjenigen, die die Entscheidungsgewalt haben, als wirtschaftlich schädigend angesehen werden. Weil dies im vorliegenden Beispiel nicht der Fall war, eskalierte der Konflikt weiter, sodass ein dritter Wirkmechanismus moralischer Argumente relevant wurde.

\subsection{Wirkmechanismen moralischer Argumente in der Öffentlichkeit}

Die Bedingungen, die moralische Argumente als Appelle an individuelle Entscheidungsträger wirken lassen, waren bei Fernlich nicht gegeben. Auch konnten moralische Argumente keinen Effekt über eine Senkung des Sozialkapitals erzielen. Sie wirkten deshalb schließlich über einen dritten Mechanismus. Arbeitnehmervertreter warfen der Geschäftsleitung öffentlich das vor, womit diese sich bisher legitimierte: Gewinnmaximierung. 
Dieser Vorwurf ist paradox. Kapitalistische Unternehmen beruhen auf der privaten, eigeninteressierten Verfügung an Produktionsmitteln und deren möglichst gewinnbringendem Einsatz. Trotzdem konnten Arbeitnehmervertreter die Öffentlichkeit gegen eben diese eigeninteressierte Verfügung an Produktionsmitteln mobilisieren und dadurch Druck aufbauen. Nicht die Gewinnerwirtschaftung selbst war es, weshalb das Unternehmen von der Öffentlichkeit angegriffen wurde; es war das Argument der Gewinnmaximierung, welches die Öffentlichkeit - vermittelt durch die Medien - aufbrachte. Die Zeitungen ignorierten, dass andere Unternehmen zur gleichen Zeit weitaus mehr Beschäftigte entließen als Fernlich; sie argumentierten stattdessen mit den Arbeitnehmervertretern gegen Fernlichs offen kommuniziertes Ziel der Gewinnmaximierung.

Nicht die Menge der Entlassungen beklagten die Medien, sondern dass eine Organisation hohe Gewinne nicht nutzte, um Arbeitsplätze zu erhalten. Zwar gibt es in der orthodoxen ökonomischen Theorie und der Shareholder-Value-Philosophie keine ökonomische Regel, wonach Gewinne Arbeitsplätze schaffen sollen (Friedman 2001). Jedoch ist diese Regel offenkundig eine gesellschaftliche Norm, deren Verletzung wirtschaftlich kostspielige Proteste nach sich zieht. Diese Proteste legen nahe, dass Gewinne nur dann als gesellschaftlich legitim darstellbar sind, wenn Unternehmen darlegen können, wie Gewinnerzielung auch der Belegschaft dient - statt lediglich den Aktionären. Den wirtschaftlich schmerzhaften Entzug öffentlicher Legitimität, den ein Unternehmen erleidet, das dies nicht darlegen kann, schilderten ein Mitglied von Fernlichs Arbeitnehmervertretung und ein Mitglied der Geschäftsleitung (in dieser Reihenfolge). Beide äußerten, dass Druck entstand, als Fernlichs öffentliche Legitimität schwand:

„Die Offiziellen der Stadt waren auf Seiten der Beschäftigten. Das ist jetzt nicht so, dass die die Gewerbesteuer raufsetzen, so simpel ist es sicherlich nicht. Aber gleichwohl, vorstellbar ist: Wie behandelt eine Gewerbeaufsicht ein Problem mit Fernlich? [...] Bis hin zu handfesten wirtschaftlichen Folgen. Es soll Fälle gegeben haben, wo Leute beim Kaufen ausdrücklich etwas verlangt haben, was nicht von Fernlich ist. [...] Da haben die Leute ohne Steuerung von sich aus gesagt: ,Jetzt will ich das Unternehmen nicht auch noch unterstützen, denn sie sind für mich moralisch diskreditiert. " (Fernlich 5. Interview: 18. Min.)

„Ich glaube, dass es tatsächlich so eine Mischung war aus öffentlichem Ansehen und: „Hoppla, wir können uns nicht leisten, dass der Kratzer, den wir jetzt haben, noch tiefer wird.' Und das noch gespiegelt nach innen. Wir kennen die latente Unruhe, die in der Belegschaft drinnen ist, weil diskutiert wird, nämlich die, die sagen: ,Ist doch alles völlig richtig, was das Unternehmen mit den Kollegen macht' und die, die sagen: ,Ich halte das für nicht richtig. ' Ich denke, da ist eine Gemengelage entstanden, wo Arbeitsdirektor Stoller völlig richtig gesagt hat: ,Einer der beiden Gründe würde schon reichen, aber beide zusammengefasst bedeuten, dass wir das Ding tatsächlich möglichst bald befrieden müssen. “ (Fernlich 3. Interview: 42. Min.; vgl. ähnlich Fernlich 2. Interview: 82. Min.)

Öffentliche Proteste gegen die Legitimität eines Unternehmens, die durch den Vorwurf der Gewinnmaximierung hervorgerufen werden, wirken somit in das jeweilige Unternehmen zurück, indem sie Wirkmechanismus zwei unterstützen: die Agitation der Belegschaft. Sie senken zudem die Bereitschaft, Produkte des Unternehmens zu konsumieren, und befördern eine kritischere staatliche Regulierung (vgl. auch King 2008, S.413). Die Wirkkette dieses Mechanismus, in dem moralische Argumente durch Agitation der 
Öffentlichkeit ihre Macht entfalten, besteht darin, dass 1) moralische Argumente die öffentliche Legitimität einer Organisation angreifen, 2) die Organisation abhängig von dieser Legitimität ist und 3) Entscheidungsträger der Organisation diese Abhängigkeit erkennen. Wenn einer dieser Aspekte nicht gegeben ist, können moralische Argumente auch nicht über diesen Wirkmechanismus greifen. Im vorliegenden Fall wirkten moralische Argumente jedoch über diesen Einflussmechanismus.

\section{Diskussion}

Unter Rückgriff auf eine Erhebung des Statistischen Bundesamtes ging dieser Artikel am Beispiel von Produktionsverlagerungen in Niedriglohnländer der Frage nach, wie und wie stark moralische Argumente wirtschaftliches Handeln beeinflussen. Unternehmen bezeichneten in der oben genutzten Umfrage moralische Bedenken als eines der größten Verlagerungshemmnisse. In der logistischen Regressionsanalyse der Daten zeigte sich, dass die Wahrscheinlichkeit zu verlagern signifikant geringer ist, wenn ein Unternehmen moralische Bedenken gegenüber einer Verlagerung äußert. Eine Fallstudie untersuchte daraufhin, wie es zu diesem Zusammenhang kommt, mittels welcher Mechanismen also moralische Argumente Verlagerungspläne beeinflussen. Drei solcher Mechanismen ließen sich herausarbeiten.

Erstens können moralische Argumente unter Ungewissheit beeinflussen, was Akteure als wirtschaftlich rational definieren (Kaplan 2008). Dies kann erklären, warum befragte Unternehmen, die eine Verlagerung nicht durchführten, diese auch für moralisch problematischer hielten: Sie sahen aufgrund moralischer Bedenken möglicherweise eher die wirtschaftlich problematischen Aspekte einer Verlagerung, während sie mögliche Vorteile in den Hintergrund rückten. Zweitens zeigte sich, dass moralische Argumente das Sozialkapital innerhalb einer Organisation verringern und dadurch Druck ausüben können, weil und insofern die Verringerung von Sozialkapital wirtschaftlich kostspielig ist. Drittens können moralische Argumente ein Unternehmen als gewinnmaximierend darstellen, was öffentliche Legitimität entzieht und das Wohlwollen der Kunden und staatlicher Regulierer beschädigt.

Mit den in der explorativen Fallstudie herausgearbeiteten Mechanismen versuchte der Artikel, eine bestehende Forschungslücke zu schließen. Während weithin argumentiert wird, dass moralische Argumente gegenüber Unternehmen unter Umständen wirken, versuchte dieser Artikel aufzuklären, unter welchen genauen Umständen dies der Fall ist und welche verallgemeinerbaren Mechanismen dabei wirksam sind. Weitergehende Forschung muss zeigen, inwiefern die hier skizzierten Mechanismen moralischer Einflussnahme auch in anderen Organisationen wirksam sind und ob sie damit erschöpfend erfasst wurden oder ob weitere Mechanismen eine Rolle spielen. Eine offene Frage ist zudem, ob und wie die hier präsentierten Einflussmechanismen institutionenabhängig sind, d. h. nur mittels und im Rahmen bestimmter Institutionen wirken. Während grundsätzlich davon auszugehen ist, dass moralische Argumente in den verschiedensten Umfeldern individuelle Rationalitätsdefinitionen von Akteuren, das Sozialkapital und die öffentliche Legitimität von Organisationen und Unternehmen beeinflussen, so ist doch anzunehmen, dass beispielsweise die Mitbestimmung in Deutschland ein vorteilhaftes institutionelles Umfeld bietet, um Geschäftsleitungen zu beeinflussen. Nicht zuletzt hängt es zudem von 
der jeweiligen Kultur und den dominierenden Vorstellungen von Leistungsgerechtigkeit $\mathrm{ab}$, wie sehr Gewinnmaximierung öffentlich als unmoralisch präsentiert werden kann.

\section{Literatur}

Abel, T. (1948). The operation called Verstehen. American Journal of Sociology, 54, 211-218.

Bauer, H., \& Hardock, P. (2003). Hemmnisse der Produktionsverlagerung von Industrieunternehmen ins Ausland. In T. Peske \& R. Schrank (Hrsg.), Strategie, Innovation und Internationalisierung (S. 261-287). Köln: Josef Eul Verlag.

Beckert, J. (1996). What is sociological about economic sociology? Uncertainty and the embeddedness of economic action. Theory and Society, 25, 803-840.

Blau, P. M. (1964). Exchange and power in social life. New York: Wiley.

Blau, P. M. (1969). The dynamics of bureaucracy. A study of interpersonal relations in two government agencies. Chicago: The University of Chicago Press.

Durkheim, E. (1953). The determination of moral facts. In E. Durkheim, Sociology and philosophy (S. 35-62). Glencoe: The Free Press.

Durkheim, E. (1977). Über soziale Arbeitsteilung. Studie über die Organisation höherer Gesellschaften. Frankfurt a. M.: Suhrkamp.

Durkheim, E. (1986). Einführung in die Moral. In H. Bertram (Hrsg.), Gesellschaftlicher Zwang und moralische Autonomie (S. 33-53). Frankfurt a. M.: Suhrkamp.

Durkheim, E. (1999). Physik der Sitten und des Rechts. Vorlesungen zur Soziologie der Moral. Frankfurt a. M.: Suhrkamp.

Eckstein, H. (1975). Case studies and theory in political science. In F. Greenstein \& N. Polby (Hrsg.), Handbook of political science (S. 79-138). Reading: Addison-Wesley.

Fourcade, M., \& Healy, K. (2007). Moral views of market society. Annual Review of Sociology, $33,285-311$.

Friedman, M. (2001). The social responsibility of business is to increase its profits. In R. Almeder, J. Humber \& M. Snoyenbos (Hrsg.), Business ethics (S. 72-78). New York: Prometheus Books.

Geertz, C. (1973). The interpretation of cultures: Selected essays. New York: Basic Books.

George, A. L., \& Bennett, A. (2005). Case studies and theory development in the social science. Cambridge: The MIT Press.

Gerring, J. (2007). Case study research. Principles and practices. Cambridge: Cambridge University Press.

Glaser, B. G., \& Strauss, A. L. (1967). The discovery of grounded theory. Strategies for qualitative research. London: Weidenfeld and Nicolson.

Gläser, J., \& Laudel, G. (2004). Experteninterviews und qualitative Inhaltsanalyse als Instrumente rekonstruierender Untersuchungen. Wiesbaden: VS Verlag für Sozialwissenschaften.

Gouldner, A. (1954). Patterns of industrial bureaucracy. New York: The Free Press.

Granovetter, M. (1985). Economic action and social structure. The problem of embeddedness. American Journal of Sociology, 91, 481-510.

Hall, P. (2002). Aligning ontology and methodology in comparative research. In J. Mahoney \& D. Rueschemeyer (Hrsg.), Comparative historical analysis in the social sciences (S. 373-404). New York: Cambridge University Press.

Hall, P. (2007). Systematic process analysis: When and how to use it. European Political Science, 7, 304-317.

Healy, K. (2004). Altruism as an organizational problem: The case of organ procurement. American Sociological Review, 69, 387-404. 
Hedström, P., \& Swedberg, R. (1998). Social mechanism: An introductory essay. In P. Hedström \& R. Swedberg (Hrsg.), Social mechanisms: An analytical approach to social theory (S. 1-31). Cambridge: Cambridge University Press.

Hiß, S. (2006). Warum übernehmen Unternehmen gesellschaftliche Verantwortung? Ein soziologischer Erklärungsversuch. Frankfurt a. M.: Campus.

Kaplan, S. (2008). Framing contests: Strategy making under uncertainty. Organization Science, 19, $729-752$.

Keck, M. E., \& Sikkink, K. (1998). Activists beyond borders. Ithaca: Cornell University Press.

King, B. G. (2008). A political mediation model of corporate response to social movement activism. Administrative Science Quarterly, 53, 395-421.

King, B. G., \& Pearce, N. A. (2010). The contentiousness of markets: Politics, social movements, and institutional change in markets. Annual Review of Sociology, 36, 249-267.

King, B. G., \& Soule, S. A. (2007). Social movements as extra-institutional entrepreneurs: The effect of protests on stock price returns. Administrative Science Quarterly, 52, 413-442.

Luders, J. (2006). The economics of movement success: Business responses to civil rights mobilization. American Journal of Sociology, 111, 963-998.

Margolis, J. D., \& Walsh, J. P. (2003). Misery loves companies: Rethinking social initiatives by business. Administrative Science Quarterly, 48, 268-305. http://www.jstor.org/stable/3556659. Zugegriffen: Jan. 2013.

Marquis, C., Glynn, M. A., \& Davis, G. F. (2007). Community isomorphism and corporate social action. Academy of Management Review, 32, 925-945.

Mayntz, R. (2002). Zur Theoriefähigkeit makro-sozialer Analysen. In R. Mayntz (Hrsg.), Akteure - Mechanismen - Modelle. Zur Theoriefähigkeit makro-sozialer Analysen (S.7-43). Frankfurt a. M.: Campus.

Putnam, R. (1993). Making democracy work. Civic traditions in modern Italy. Princeton: Princeton University Press.

Schütz, A. (1971). Gesammelte Aufsätze I. Das Problem der sozialen Wirklichkeit. Den Haag: Nijhoff.

Shamir, R. (2008). The age of responsibilization: On market-embedded morality. Economy and Society, 37, 1-19.

Soule, S. A. (2009). Contention and corporate social responsibility. New York: Cambridge University Press.

Statistisches Bundesamt. (2008). Verlagerung wirtschaftlicher Aktivitäten. Ergebnisse der Piloterhebung. Wiesbaden: Statistisches Bundesamt.

Strauss, A. L., \& Corbin, J. (1990). Basics of qualitative research. Grounded theory procedures and techniques. Newbury Park: Sage.

Vaara, E., Tienari, J., \& Laurila, J. (2006). Pulp and paper fiction: On the discursive legitimation of global industrial restructuring. Organization Studies, 27, 789-810.

Weber, M. (1980). Wirtschaft und Gesellschaft. Grundriß der verstehenden Soziologie. Tübingen: J.C.B. Mohr.

Weber, M. (1988). Gesammelte Aufsätze zur Religionssoziologie I. Tübingen: J. C. B. Mohr.

Zwania, J. (2008). Verlagerung wirtschaftlicher Aktivitäten. Theoretischer Hintergrund zur Erhebung. Wirtschaft und Statistik, 6/2008, 477-482.

Martin Schröder, geb. 1981. Dr., Juniorprofessor an der Philipps-Universität Marburg. Forschungsschwerpunkte: Einfluss moralischer Argumente auf wirtschaftliches Handeln und soziale Ungleichheit; Kapitalismusvarianten und Wohlfahrtsstaaten. Ausgewählte Veröffentlichungen: Integrating welfare and production typologies. How refinements of the varieties of capitalism approach call for a combination with welfare typologies, in: Journal of Social Policy, 2009; Die Macht moralischer Argumente. Produktionsverlagerungen zwischen wirtschaftlichen Interessen und gesellschaftlicher Verantwortung, 2011; Integrating varieties of capitalism and welfare state research: A unified typology of capitalisms, 2013. 